\title{
Solução Interativa Fuzzy para o Modelo de Crescimento de Organismos Procariontes
}

\author{
Sílvia D. Souza ${ }^{1}$ \\ DM/UFAM, Manaus, AM \\ Roberto A. C. Prata ${ }^{2}$ \\ DM/UFAM, Manaus, AM \\ Laécio C. de Barros ${ }^{3}$ \\ IMECC/UNICAMP, São Paulo, SP
}

\begin{abstract}
Resumo. O objetivo deste trabalho é propor um modelo matemático fuzzy baseado no modelo de Loladze, no qual se modela o crescimento de seres procariontes e eucariontes. Estudamos o equilíbrio de dois processos fundamentais,(i) a síntese de proteína e(ii) síntese de rRNA(ribossômico) de organismos procariontes em que o resultado é um atrator bioquímico estável que produz homeostaticamente uma dada proporção de proteína. Inicialmente, encontramos a solução fuzzy para o modelo proposto por Loladze, em que a taxa de proteína por unidade de massa foi considerada fuzzy, pois na decomposição biomolecular alguns parâmetros sofrem variação com pequenas variações de temperatura. Por fim, foi determinada a solução interativa fuzzy que correlaciona a taxa de proteína por unidade de massa e a taxa de síntese de rRNA por unidade de massa proteica.

Palavras-chave. Seres Eucariontes, Números Fuzzy Interativos, Princípio de Extensão de Zadeh, Biomatemática.
\end{abstract}

\section{Introdução}

A célula é definida como a unidade básica estrutural e funcional de todos os organismos vivos, unidade esta que pode se reproduzir. As células podem ser classificadas em dois grupos: procariontes ou eucariontes. A principal diferença entre ambos é a existência ou não de uma organela que contém o DNA da célula. Quando esta estrutura está presente, diz-se que a célula possui núcleo e que, portanto, é uma célula eucariótica. Caso contrário, a célula é procariótica [2].

Neste trabalho faremos um estudo sobre o ser procarionte Escherichia coli que de acordo com [9] é um bacilo gram-negativo, anaeróbico facultativo, predominante na microbiota normal do intestino humano e de outros animais. As cepas de E.coli presentes nas infecções intestinais são denominadas de E.coli diarreiogênicas (DEC) e sua classificação se dá através de soros grupos O e O:H. As DEC são classificadas em seis categorias: E.coli enteropatogênica (EPEC), E.coli enterotoxigênica (ETEC), E.coli entero-hemorrágica (EHEC), E.coli enteroinvasora (EIEC), E.coli enteroagregativa (EAEC) e a E.coli que adere difusamente (DAEC).

No presente estudo, estamos propondo um modelo matemático fuzzy baseado em [7], relacionando os parâmetros de crescimento de massa proteica $\gamma$ e de massa de rRNA $\psi$ via interatividade devido a decomposição biomolecular existente entre estes parâmetros, trazemos a solução fuzzy do modelo via extensão de Zadeh e a solução interativa fuzzy para o crescimento da E.coli a partir dos dados fornecidos em [7].

\footnotetext{
${ }^{1}$ silviadss@gmail.com

2 praroberto@gmail.com

${ }^{3}$ laeciocb@ime.unicamp.br
} 


\section{Modelo}

O modelo descrito por Loladze [7], estuda o crescimento de seres procariontes e eucariontes e busca encontrar o equilíbrio deste crescimento. Para o modelo, supomos nas condições ideais, que as células dos organismos procariontes e eucariontes cresçem exponencialmente.

Denotando por $\mathrm{m}$ a massa de todas as proteínas e por $\mathbf{r}$ a massa de todos rRNAs, expressamos a síntese de proteína por $\frac{d m}{d t}=\gamma m$ e a síntese total de rRNA por $\frac{d r}{d t}=\psi r$, onde $\gamma$ e $\psi$ são respectivamente a taxa de síntese de proteína por unidade de massa e a taxa de síntese de proteína de rRNA por unidade de massa proteica.

Para o estudo do crescimento dos seres eucariontes e procariontes, calculamos a proporção de proteína por rRNA, a qual pode ser escrita por $\beta=\frac{m}{r}$, onde $m$ e $r$ crescem exponencialmente e a razão $\frac{m}{r}$ é governada pela seguinte equação diferencial:

$$
\frac{d \beta}{d t}=\gamma-\psi \beta^{2}
$$

Ao resolver a equação $\frac{d \beta}{d t}=0$, encontra-se um equilíbrio assintoticamente estável para $\beta$. Observa-se ainda que isto independe da condição inicial e $\beta$ tende a um valor único.

Ressalta-se que, quando a massa proteica e a massa de rRNA estão na proporção $\beta=\frac{m}{r}$, as novas substâncias sintetizadas estão na mesma proporção da biomassa existente e isto equivale a um crescimento equilibrado.

\subsection{Decomposição em Componentes Biomoleculares}

Nesta seção, apresentamos os componentes biomoleculares dos parâmetros $\gamma$ e $\psi$ : $l_{a}$-Comprimento de aminoácido de RNA polimerase aapol $^{-1}$;

$l_{r}$-Comprimento de um rRNA em um ribossomo $n t r i b^{-1}$;

$m_{a}$-Média das massas de aminoácidos nas cadeias peptídicas $D a a a^{-1}$; $m_{r}$-Média das massas dos ribonucleicos Dant $^{-1}$;

$\sigma_{a}$ Taxa de alongamento peptídico $a a s^{-1} \mathrm{rib}^{-1}$

$\sigma_{r}$-Taxa de nascimento de $\mathrm{rRN} n t s^{-1} \mathrm{pol}^{-1}$;

$\phi_{a^{-}}$Fração de ribossomos que são ativamente traduzidos $m g m g^{-1}$;

$\phi_{r^{-}}$Fração total de proteínas de RNA polimerase ativamente traduzida $m g m g^{-1}$.

De acordo com [7], para o parâmetro $\gamma$, um ribossomo ativo (isto é, aquele que está ligado a uma cadeia peptídica crescente) sintetiza proteínas a uma taxa igual à taxa do alongamento da cadeia peptídica $\left(\sigma_{a}\right)$ vezes a massa média de um resíduo de um aminoácido $\left(m_{a}\right)$, isto é, $\sigma_{a} m_{a}$. Já a massa de rRNA em um ribossomo é igual à massa média de um ribonucleico $\left(m_{r}\right)$ vezes o comprimento de rRNA denotado por $l_{r}$, que mede um ribonucleico, ou seja, $m_{r} l_{r}$.

Desta forma, a taxa de síntese de proteína por unidade de rRNA é dada por $\frac{\sigma_{a} m_{a}}{m_{r} l_{r}}$. Agora, multiplicando esta fração de ribossomo ativamente traduzido por $\phi_{r}$, obtemos:

$$
\gamma=\phi_{a} \frac{\sigma_{a} m_{a}}{m_{r} l_{r}}
$$

Para determinarmos $\psi$, notamos que um RNA ativo na polimerase sintetiza o rRNA a uma taxa igual ao raio de alongamento $\sigma_{r}$ inicial vezes $m_{r}$, isto é, $\sigma_{r} m_{r}$. A massa de RNA polimerase é o produto de seu comprimento $\left(l_{a}\right)$ por $m_{a}$, isto é, $m_{a} l_{a}$. Portanto, a taxa de síntese de rRNA por unidade de RNA polimerase é dada pela razão $\frac{\sigma_{a} m_{r}}{m_{a} l_{a}}$. Assim, multiplicando a fração por $\phi_{r}$, obtemos:

$$
\psi=\phi_{r} \frac{\sigma_{r} m_{r}}{m_{a} l_{a}}
$$


Observamos que, pelas equações (2) e (3) podemos obter a seguinte relação entre as taxas de crescimento do seres procariontes e eucariontes:

$$
\psi=\frac{c_{1}}{\gamma}
$$

em que $c_{1}=\frac{\phi_{r} \sigma_{r} \phi_{a} \sigma_{a}}{l_{r} l_{a}}$.

\section{Solução Analítica}

Nesta seção, apresentamos a solução determinística do modelo.

Para $K$ e $c$ constantes reais, a solução da equação (1) é dada por:

$$
\beta(t)=\sqrt{\frac{\gamma}{\psi}}+\frac{1}{(K+c \psi) e^{2 \psi \sqrt{\frac{\gamma}{\psi}} t}-\frac{1}{2} \sqrt{\frac{\psi}{\gamma}}} .
$$

Para a representação gráfica da solução determinística, utilizamos os valores das tabelas 1 e 2 do artigo [7], onde encontramos os valores dos componentes biomoleculares que compõem as taxas de crescimento $\left(\gamma\right.$ e $\psi$ ) da bactéria E. Coli a $37^{\circ} \mathrm{C}, l_{a}=3400$ aapol $^{-1}, l_{r}=4560$ ntrib $^{-1}, m_{a}=110$ Daaa $^{-1}, m_{r}=340$ Dant $^{-1}, \sigma_{a}=21$ aas $^{-1}$ rib $^{-1}, \sigma_{r}=85 \mathrm{nts}^{-1} \mathrm{pol}^{-1}, \phi_{a}=0.80 \mathrm{mgmg}^{-1}$ e $\phi_{r}=0.0020$ $m g m g^{-1}$

Agora, substituindo os valores acima nas equações (2) e (3), obtemos os respectivos valores para as taxas de crescimento da E.Coli:

$$
\gamma=0,0012 \text { e } \psi=0,657 .
$$

O gráfico da solução determinística, representado pela Figura 1, foi gerado para os valores de $K=1$ e $c=-1$.

\subsection{Solução fuzzy}

A ideia para o estudo da solução fuzzy parte do príncipio que alguns dos componentes biomoleculares, como por exemplo $\phi$ e $\sigma$, na polimerase sofrem alterações no crescimento com pequenas variações de temperatura ( $[6])$.

O gráfico da solução fuzzy via princípio de extensão de Zadeh, quando consideramos o parâmetro $\gamma$ dado por:

$$
\gamma=(0.0011990 .00120 .001201)
$$

pode ser vizualizado na Figura 2. Observe ainda que, na simulação, utilizamos os valores oriundos da decomposição biomolecular de cada taxa de crescimento.

\subsection{Interatividade entre números fuzzy}

Nesta seção, apresentamos os conceitos introdutórios sobre a interatividade entre números fuzzy.

Definição 1. Uma distribuição de possibilidade sobre $\mathbb{R}^{n}$ é um conjunto fuzzy $C$ de $\mathbb{R}^{n}$ com função de pertinência $\mu_{C}: \mathbb{R}^{n} \longrightarrow[0,1]$ satisfazendo $\mu_{C}\left(x_{0}\right)=1$ para algum $x_{0} \in \mathbb{R}^{n}$. 


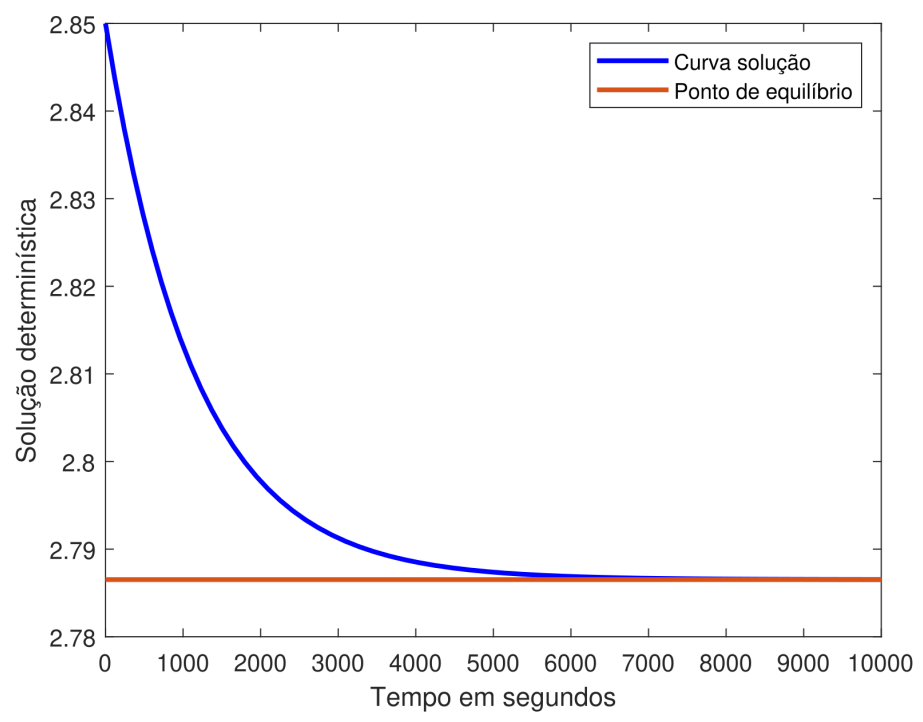

Figura 1: Gráfico da Solução Determinística

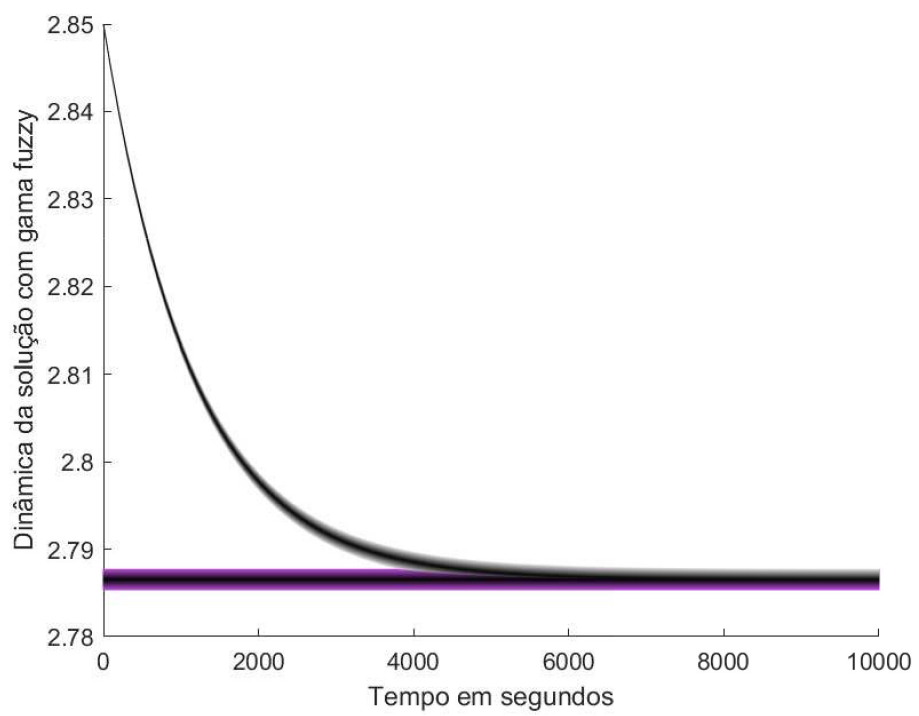

Figura 2: O Gráfico da Solução Fuzzy

A família das distribuições de possibilidade de $\mathbb{R}^{n}$ será denotada por $\mathcal{F}_{c}\left(\mathbb{R}^{n}\right)$.

Definição 2. Considere $A_{1}, \ldots, A_{n} \in \mathcal{E}(\mathbb{R})$ então $C \in \mathcal{F}_{c}\left(\mathbb{R}^{n}\right)$ é chamada uma distribuição de possibilidade conjunta se $\max _{x_{j} \in \mathbb{R}, j \neq i} \mu_{C}\left(x_{1}, \ldots, x_{n}\right)=\mu_{A}\left(x_{i}\right)$. Além disso, $A_{1}, \ldots, A_{n}$ são chamados as distribuições marginais de $C$.

Neste caso,

$\mu_{C}\left(x_{1}, \ldots, x_{n}\right) \leq \min \left(\mu_{A_{1}}\left(x_{1}\right), \ldots, \mu_{A_{n}}\left(x_{n}\right)\right)$ e $[C]^{\alpha} \subseteq\left[A_{1}\right]^{\alpha} \times \ldots \times\left[A_{n}\right]^{\alpha}$. 
Definição 3. Dois números fuzzy $A$ e $B$ são ditos não interativos se, e somente se, sua distribuição de possibilidade conjunta $C$ for dada por

$$
\mu_{C}(x, y)=\min \left\{\mu_{A}(x), \mu_{B}(x)\right\} .
$$

Caso contrário, são ditos interativos.

Para números fuzzy não interativos temos:

$$
[C]^{\alpha}=[A]^{\alpha} \times[B]^{\alpha},
$$

para todo $x \in \mathbb{R}^{n}, y \in \mathbb{R}^{m}$ e todo $\alpha \in[0,1]$.

Definição 4. Sejam $f: \mathbb{R}^{n} \longrightarrow \mathbb{R}^{k}$ uma função, $A_{1}, \ldots, A_{n}$ números fuzzy interativos com distribuição de possibilidade conjunta $C$. A extensão de $f$ aplicada a $\left(A_{1}, \ldots, A_{n}\right)$ segundo $C$ é o subconjunto fuzzy $f_{C}\left(A_{1}, \ldots, A_{n}\right)$ cuja função de pertinência é definida por

$$
\mu_{f_{C}\left(A_{1}, \ldots, A_{n}\right)}(y)=\left\{\begin{array}{ccc}
\sup _{\left(x_{1}, \ldots, x_{n}\right) \in f^{-1}(y)} \mu_{C}\left(\mu_{A_{1}}\left(x_{1}\right), \ldots, \mu_{A_{n}}\left(x_{n}\right)\right), & \text { se } & f^{-1}(y) \neq \emptyset \\
0 \quad \text { se } & f^{-1}(y)=\emptyset,
\end{array}\right.
$$

onde $f^{-1}(y)=\left\{\left(x_{1}, \ldots, x_{n}\right): f\left(x_{1}, \ldots, x_{n}\right)=y\right\}$.

Com relação ao princípio de extensão segundo $C$ ( [1]), temos:

Proposição 3.1. Sejam $A_{1}, \ldots, A_{n}$ números fuzzy, $C$ uma distribuição de possibilidade conjunta com distribuições marginais $A_{1}, \ldots, A_{n}$ e $f: \mathbb{R}^{n} \longrightarrow \mathbb{R}$ uma função contínua. Então

$$
\left[f_{C}\left(A_{1}, \ldots, A_{n}\right)\right]^{\alpha}=f\left([C]^{\alpha}\right),
$$

para todo $\alpha \in[0,1]$. Além disso, $f_{C}\left(A_{1}, A_{2}, \ldots, A_{n}\right)$ é sempre um número fuzzy.

Definição 5. Sejam $f: X \longrightarrow Y, X, Y \subset \mathbb{R}$ uma função monótona injetora e $A, B \in \mathcal{E}(\mathbb{R})$ números fuzzy. Dizemos que $A$ e $B$ são correlacionados segundo a função $f$ ou f-correlacionados, se sua distribuição de possibilidade conjunta $C$ é dada por:

$$
\mu_{C}(x, y)=\mu_{A}(x) \mathcal{X}_{\{y=f(x)\}}(x, y)=\mu_{B}(y) \mathcal{X}_{\{y=f(x)\}}(x, y)
$$

onde,

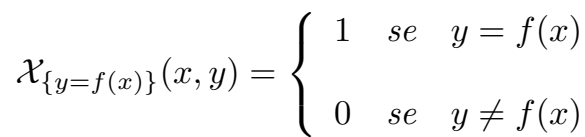

é a função característica da curva $\gamma(x)=(x, f(x))$.

Neste caso, temos:

$$
\begin{aligned}
& {[B]^{\alpha}=f\left([A]^{\alpha}\right), \forall \alpha \in[0,1],} \\
& \begin{aligned}
\mu_{B}(x)=\mu_{A}\left(f^{-1}(x)\right), \forall x \in \mathbb{R} & \text { se }[A]^{\alpha}=\left[a_{1}^{\alpha}, a_{2}^{\alpha}\right], \\
& =\left\{(x, y) \in \mathbb{R}^{2}: \mu_{C}(x, y) \geq \alpha\right\} \\
& =\left\{(x, y) \in \mathbb{R}^{2}: \mu_{A}(x) \mathcal{X}_{\{y=f(x)\}}(x, y) \geq \alpha\right\} \\
& =\left\{(x, f(x)): \mu_{A}(x) \geq \alpha\right\} \\
& =\left\{(x, f(x)): x \in\left[a_{1}^{\alpha}, a_{2}^{\alpha}\right]\right\} .
\end{aligned}
\end{aligned}
$$

Quando $f(x)=\frac{q}{x}+r$, com $x>0$ e $q \neq 0$, os números fuzzy $A$ e $B$ são chamados hiperbolicamente correlacionados $([3])$. 


\subsection{Solução Interativa Fuzzy para o Modelo}

De acordo com [8] et al, os modelos matemáticos para fenômenos biológicos contém incertezas, tanto nas suas variáveis de estado quanto nos parâmetros das equações dos modelos.

Neste trabalho, estamos propondo um estudo através de interatividade para o modelo (1), onde as variáveis são hiperbolicamente correlacionadas e para a obtenção dos resultados utilizamos os dados encontrados em [7].

A solução interativa fuzzy do modelo (1), onde $\gamma$ e $\psi$ são hiperbolicamente correlacionados, é descrita abaixo:

Para a equação do modelo (1), $\gamma$ é modelado pelo número fuzzy triangular:

$$
\gamma=\left(\begin{array}{lll}
0.001199 & 0.0012 & 0.001201
\end{array}\right)
$$

$\mathrm{Ou}$, equivalentemente,

$$
[\gamma]^{\alpha}=[0.00001 \alpha+0.001199,0.00129 \alpha-0.001201], \quad \alpha \in[0,1]
$$

Pela equação (3), a taxa de crescimento dos rRNA pode ser representada pelo seguinte número fuzzy:

$$
[\psi]^{\alpha}=\left[\frac{c_{1}}{0.00129-0.00009 \alpha}, \frac{c_{1}}{0.000001 \alpha+0.001199}\right]
$$

Agora, aplicando os conceitos encontrados em [4] e [5], obtemos a distribuição de possibilidade conjunta $[J]^{\alpha}$ entre os parâmetros $\gamma$ e $\psi$ :

$$
\mu_{J}(\gamma, \psi)=\mu_{\psi}(\gamma) \mathcal{X}_{\left.\left\{\psi=\frac{1}{\gamma}\right)\right\}}(\gamma, \psi)
$$

Segue que a solução interativa fuzzy, em que os parâmetros $\gamma$ e $\psi$ são hiperbolicamente correlacionados, dada por:

$$
\left[\left(\beta_{t}\right)_{J}(\gamma, \psi)\right]^{\alpha}=\left(\beta_{t}\right)\left([J]^{\alpha}\right)=\left\{\beta_{t}(\gamma, \psi):(\gamma, \psi) \in[J]^{\alpha}\right\}=\frac{\gamma}{\sqrt{c_{1}}}+\frac{1}{\left(K+C \frac{c_{1}}{\gamma}\right) e^{2 \sqrt{c} t}-\frac{1}{2} \frac{\sqrt{c}_{1}}{\gamma}} ; \gamma \in[\gamma]^{\alpha}
$$

\section{Conclusões}

Neste trabalho, apresentamos a solução fuzzy do modelo determinístico, via princípio de extensão de Zadeh da solução determinística. Desta forma, consideramos a taxa de crescimento das massas de proteína de organismos procariontes fuzzy $(\gamma)$ devido decomposição dos componentes biomoleculares que sofrem variação quando não estão na condição ideal de $37^{0} \mathrm{C}$.

Ao propormos a solução fuzzy do modelo determinístico (1), temos um resultado mais realista, visto que, o crescimento da bactéria E.coli dificilmente se dá em condição ideais. Ao modelarmos as variáveis do parâmetro $\gamma$, usando lógica fuzzy, contemplamos uma faixa de soluções que reflete estas possíveis variações e, por fim, obtivemos a solução interativa fuzzy que correlaciona fuzzy hiperbolicamente as taxas das massas de proteína e dos rRNA de organismos procariontes e pode nos auxiliar futuramente no cálculo do índice de correlação entre estas taxas. 


\section{Referências}

[1] Barros, L. C., Bassanezi, R. C. and Lodwick, W. A. First Course in Fuzzy Logic, Fuzzy Dynamical Systems and Biomathematics. Springer. Berlin Heidelberg, 2016.

[2] Brock, T. D., Madigan, M. T., Martinko, J. M., Parker, J. Biology of Microorganisms, Prentice-Hall, volume 82, page 47-49, 1994.

[3] Cabral, V. M., Prata, R. A. C. and Barros, L. C. f-correlated fuzzy numbers applied to HIV model with protease inhibitor therary, Mathware and Solt Computing Magazine, volume 22, page 46-64, 2015.

[4] Carlsson, C., Fúller, R. and Majlender, P. On possibilistic correlation, Fuzzy Sets and Systems, volume 155, page 425-445, 2006.

[5] Carlsson, C., Fúller, R. and Majlender, P. On interactive fuzzy Numbers, Fuzzy Sets and Systems, volume 143, page 355-369, 2004.

[6] Karpinets, T. V., Greenwood, D. J., Sams,. C. E . and Ammons, J .T. RNA protein ratio of the unicellular organism as a characteristic of phosphoous and nitrogen stoichiometry and the cellular requirement of ribosomes for protein synthesis, BMC, 2006.

[7] Loladze, I. and Elser, J. J. The origins of the Redfield nitrogen-to-phosphorus ratio are in a homoeostatic protein-to-rRNA ratio, Ecology Letters, volume 14, page 244-250, 2011.

[8] Massad, E., Ortega N. R. S, Barros, L. C. and Struchiner, C. J., Fuzzy Logic in action: Applications in epidemiology and beyond. Springer Science e Business, 2009.

[9] Nataro, J. P. and Kaper, B. Diarrheagenic Escherichia coli, Clin Microbiol Rev, volume 11, page 42-201, 1998. 